\title{
Using Coverage for Measuring the Effect of Haptic Feedback in Human Robotic Swarm Interaction
}

\author{
Steven Nunnally ${ }^{1}$, Phillip Walker ${ }^{1}$, Nilanjan Chakraborty ${ }^{2}$, Michael Lewis ${ }^{1}$ and Katia Sycara ${ }^{2}$
}

\begin{abstract}
A robotic swarm is a decentralized group of robots which overcome failure of individual robots with robust emergent behaviors based on local interactions. These behaviors are not well built for accomplishing complex tasks, however, because of the changing assumptions required in various applications and environments. A new movement in the research field is to add human input to influence the swarm in order to help make the robots goal directed and overcome these problems. This research in Human Swarm Interaction (HSI) focuses on different control laws and ways to integrate the human intent with local control laws of the robots. Previous studies have all used visual feedback through a computer interface to give the user the swarm state information. This study adapted swarm control algorithms to give the operator haptic feedback as well as visual feedback. The study shows the benefits of the additional feedback in a target searching class. Researchers in multi-robot systems have shown benefits of haptic feedback in obstacle navigation before, but this study is a novel method because of the decentralized formation of the robotic swarm. In most environments, operators were able to cover significantly more area, increasing the chance of finding more targets. The other environment found no significant difference, showing that the haptic feedback does not degrade performance in any of the tested environments. This supports our hypothesis that haptic feedback is useful in HSI and requires further research to maximize its potential.
\end{abstract}

\section{INTRODUCTION}

Swarm robotics use simple robots with local control laws in large numbers to create emergent behaviors generally robust to failure of individuals. Limited sensing and computational power is a general assumption for swarm robotics because of the cost necessary to produce large groups that can accomplish tasks efficiently. These emergent behaviors could be very useful in applications such as search and rescue and military surveillance, but generally the algorithms are only guaranteed in very controlled environments. Therefore, these real world applications require a need for human assistance to help influence the swarm to become more goal directed in complex various and changing environments [1], [2], [3], [4], [5].

Previous studies in Human Swarm Interaction (HSI) focus on the control laws and means for an operator to exert influence on the swarm [6], [7], [1], [2], [3], [8], [5]. Few studies show results of user studies and only show a proof of concept with a few real robots [9], [1]. Others have

\footnotetext{
*This research has been sponsored in part by AFOSR FA955008-10356 and ONR Grant N0001409-10680

${ }^{1}$ School of Information Science, University of Pittsburgh, Pittsburgh, PA 15213, USA smn34@pitt.edu, pmwalk@gmail.com, mlesis.pitt.edu

${ }^{2}$ Robotics Institute, Carnegie Mellon University, Pittsburgh, PA 15213, USA nilanjan@cs.cmu.edu, katia@cs.cmu.edu
}

built theories and models around a hybrid system requiring a human operator and autonomous algorithm to show the downfalls and design challenges that must be considered when creating new control laws for HSI [10], [1], [11]. Other studies various levels of user studies, ranging from only a few creators showing results to numerous new users testing the system [6], [7], [2], [3], [4], [5], [11]. These studies use simulation and analyze the operators ability to use the given control laws to influence the swarm in the completion of some task, like information foraging or radiation detection.

No study listed above considers passing the swarms state back to the operator through any other channel besides the visual channel. The haptic channel has been considered in multi-robot systems and has shown success in navigation of obstacle filled environments [12], [13], [14], [15]. These studies rely on robots to maintain rigid spatial relations and precise sensing and communication to deliver tactile information about approaching obstacles or deviation from a planned path through the input device of the operator. The researchers were also able to overcome communication delays between the robots' sensing information and the haptic input device with simulation tests on a few robots [12], [15]. The manner in which the feedback was generated and given to the operator would not scale well because of the rigidity of the formation and the treatment of the system as a rigid structure of robots, who act upon the environment as if they were one unit. The haptic force returned to the operator is based on this formation, as if it was one sensing robot and not a fluid group. Therefore, there is a need to explore using haptic feedback in robot swarm systems where the connection topology is flexible and the group size must be scalable. This is the contribution of this study.

This study creates a novel control algorithm for a swarm of robots based on a vector field algorithm modified from [16]. Each robot determines its motion based on attractive and repellant forces between other robots and obstacles. These repellant forces from obstacles afford an aggregated haptic feedback across all robots so operators can efficiently direct the swarm. This is a novel method of using haptic feedback that can work with a decentralized group of swarms without a rigid formation. The study looks to see if the availability of aggregated haptic feedback can help the operator explore an environment looking for targets. The individual robots of the system can only sense obstacles, robots, and targets with a limited sensing radius. The task is specified at a high level of searching the environment to find and mark targets, so that the operator is forced to make the swarm goal directed and cover as much area as possible. Operators 

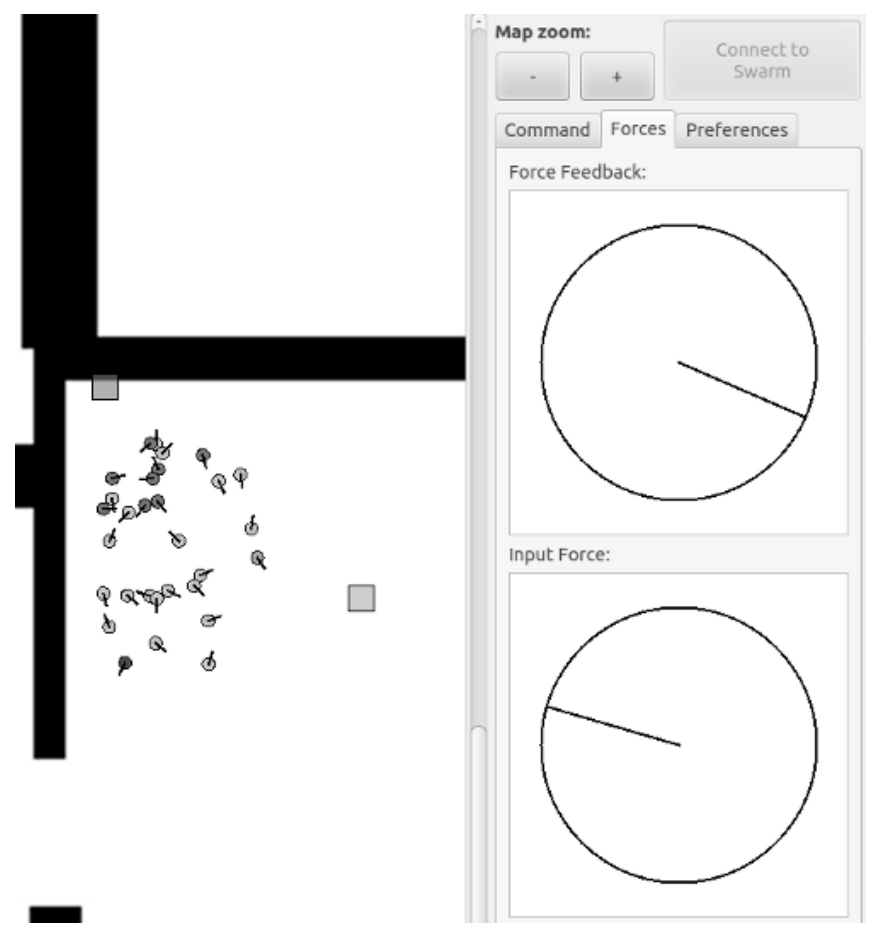

Fig. 1. The GUI used for every condition of the study. The left side shows the robots' estimated positions, obstacles, and marked targets. The right side shows the Force Feedback panel calculated in 2, and the Input Force panel calculated in 4 .

are asked to explore four obstacle-filled environments with different features to find and mark targets. We use a betweensubjects design for the user study, so that half of the users receive haptic feedback along with all of the visual feedback that the other group receives. Each group receives visual information about the forces acting upon the swarm, but it is our hypothesis that the operators with haptic feedback will better comprehend the swarms state and better convey intent to maximize their performance for the given task, even though the feedback is aggregated across the group. This paper extends data analysis from a previous study which failed to find significant improvements with the number of targets found between the operator groups [17]. Instead, environment coverage is used as a metric for performance to remove the luck factor of finding and marking targets. The experiment, interface, and swarm algorithm is explained in section II. Section III presents the results of the user study. Finally, the findings and future works of the study are presented in section IV.

\section{EXPERIMENT}

\section{A. Interface}

The environment, targets, and swarm consisting of 30 differential drive P2AT robots is simulated in Stage v. 3.2.2 [18]. The graphical interface and robot control laws are implemented using the Robot Operating System (ROS) [19], see Fig. 1. The robots positions within the environment are updated on screen with a bird's eye view. Operators can scroll and zoom this view using a mouse as needed to complete

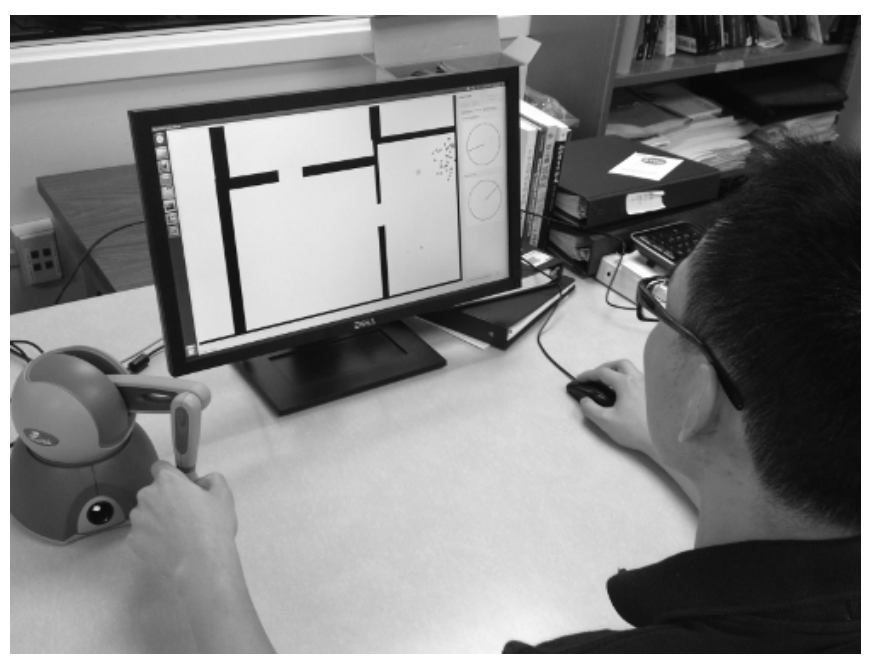

Fig. 2. Participants used an Omni Phantom device (left) and mouse to influence the swarm in the interface.

the task. The robots are represented as circles with lines pointing in the direction of their heading. When a robot senses a target, the color of the robot changes on the display to match the color of the target, so that the operator knows a target is nearby. A target is only marked on the interface and considered found when a threshold of five robots sense the target simultaneously. Obstacles are shown in the interface in all but one of the environments. They appear as black rectangles, with traversable areas in white. A Phantom Omni device is used as the input and haptic feedback output device, see Fig. 2. The device's coordinate frame is centered on a circle on the desktop so the user can create forces to influence the swarm's motion by moving the end effector around in that circle. On the screen, the Input Force panel shows this force representation next to the robot viewing panel so that operators do not have to look down at the input device to determine their influence. The Force Feedback panel, found above the Input Force panel, shows feedback of the obstacle forces averaged across the swarm as a whole and matches the haptic feedback for those operators who are in that condition. This aggregation might muddle the swarms state and it's interaction with the environment, but should still aide the operator in exploring the environment. The forces in these panels and their uses in the system are described in the next subsection. All of the forces, robot positioning data, and targeting marking were logged every second for data analysis purposes.

\section{B. Robot Algorithms}

This study presents novel control laws for swarm based on the vector field algorithm used in [16], which uses repulsive forces from other robots and obstacles in order to deploy robots covering as much area as possible for a target searching task. The potential field for the force acting on a robot is given by:

$$
\mathbf{F}=\mathbf{F}_{o}+\mathbf{F}_{r}+\mathbf{F}_{h}
$$




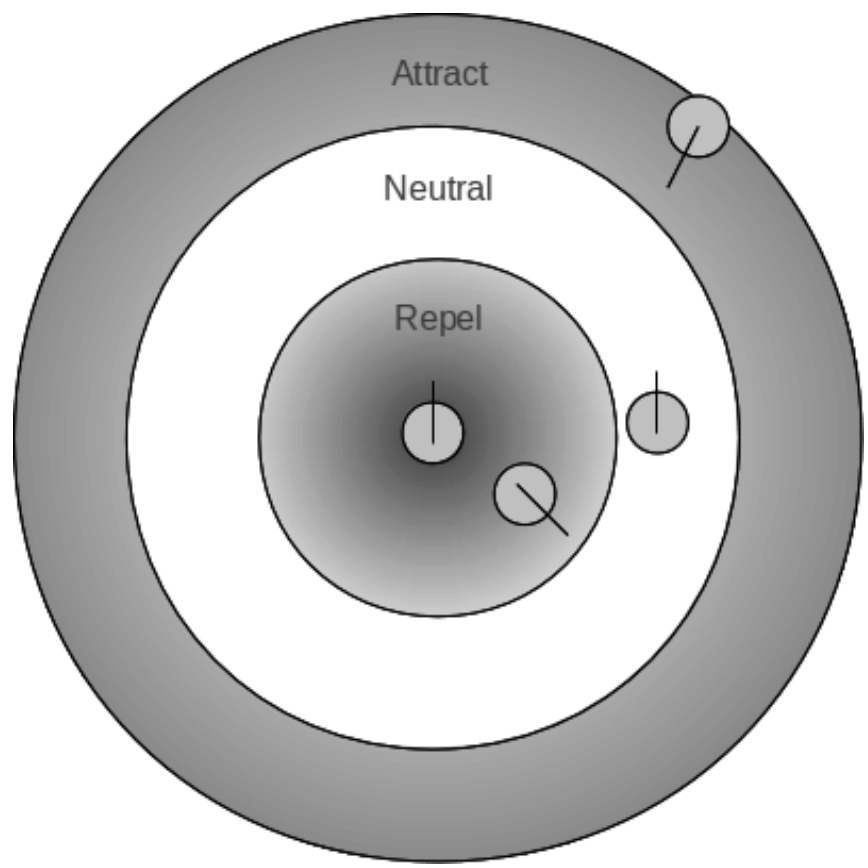

Fig. 3. The closer the robots are, the stronger the repulsive force, the further the robots are, the strong the attractive force. The robots stabilize to the neutral zone without $\mathbf{F}_{\mathbf{o}}$ and $\mathbf{F}_{\mathbf{h}}$.

with the forces due to obstacles, robots, and human influence respectively. More precisely, let $o \in V\left(q_{i}\right)$ be all obstacles in range of the robot located at $q_{i}$ and $r_{o}=$ $\left|q_{i}-q(o)\right|$ be the absolute distance between the obstacle and the robot and $\mathbf{r}_{o}=q_{i}-q(o)$ be the vector between the robot and the obstacle, then:

$$
\mathbf{F}_{o}=-k_{o} \sum o \in V\left(q_{i}\right) \frac{1}{r_{o}^{2}} \frac{\mathbf{r}_{\mathbf{o}}}{r_{o}}
$$

The average of this force across all robots represents the force feedback described above in the interface and the haptic device. Similarly, let $r \in V\left(q_{i}\right)$ be all robots in range, $r_{i}$, of the robot located at $q_{i}$ and $\mathbf{r}_{r}=\left|q_{i}-q(r)\right|$ be the distance between the robots and $\mathbf{r}_{r}=q_{i}-q(r)$ be the vector between the robots and $n_{b}$ be the beginning radius of the neutral zone and $n_{e}$ be the ending radius of the neutral zone, then:

$$
\mathbf{F}_{r}= \begin{cases}-k_{r} \sum_{r \in V\left(q_{i}\right) \frac{1}{r_{r}^{2}} \frac{\mathbf{r}_{\mathbf{r}}}{r_{r}}} & \text { if } q_{i}<n_{b} \\ k_{r} \sum_{r \in V\left(q_{i}\right) \frac{1}{\left(r_{i}-r_{r}\right)^{2}} \frac{\mathbf{r}_{r}}{r_{i}-r_{r}}} & \text { if } q_{i}>n_{e}\end{cases}
$$

This is used to disperse the robots in a controlled manner, while the attraction dissuades the robots from leaving the limited sensing range of 4 meters to maintain a more cohesive swarm to redundantly cover area so the threshold number of robots can mark targets, see Fig. 3. Finally, let $h$ be the input force vector from the Phantom device and $r_{h}=|h|$ be the magnitude of this vector and $\mathbf{r}_{h}=h$ and $h_{\text {max }}$ be the magnitude of the maximum allowed force, then:

$$
\mathbf{F}_{h}=k_{h} \frac{1}{\left(h_{\max }-r_{h}\right)^{2}} \frac{\mathbf{r}_{h}}{h_{\max }-r_{h}}
$$

This force moves the robot in the general direction that the operator applies, although the operator's control is only considered an influence because of the obstacle and other robot forces are still applied to each individual. For this study, the values assigned to the constants are $k_{o}=4$, $k_{r}=2, k_{h}=5, n_{b}=2.0, n_{e}=2.5, h_{\max }=4$. The $\max$ speed is $0.5 \mathrm{~m} / \mathrm{s}$, but the speed equals the magnitude of the force if it is less than 0.5. The robot senses robot and obstacle vectors with a 360 degree field of view and 4 degree resolution. The average $\mathbf{F}_{o}$ across all robots is shown in the Feedback panel in Fig. 1 and felt in the Phantom device if the participant is in the haptics condition.

The robots' target sensors are faulty and miss at a rate of $p_{n}$, where:

$$
p_{n}=(1-(r-d))^{\alpha}
$$

where $d$ is the distance to the object, $r$ is the range of the sensor, and $\alpha$ is the decay rate set to 4 . The sensor also has a chance of reporting a false positive. Occurrences of false positives were recalculated at an interval $t_{r}$ equal to some randomly sampled value between 6 and 10 seconds for each of the 30 robots. When a false positive occurred, an imaginary target was reported at a randomly chosen position at the edge of the target sensor's range. The automated target marking system only marks a target, when five robots simultaneously sense a target, using redundancy to eliminate the faulty sensor error. This faulty sensor model is created to add a secondary goal of the users to maintain a connected swarm and to create a more realistic scenario based on the limited capability assumption of robots in large swarms.

\section{Environments}

The participants worked to find as many targets as possible in four different obstacle filled environments. The starting position of the robots was in the bottom right corner.

The Math environment is a corridor maze, see Figure 4(a). The width of the halls was such that the robots could mark targets along both of the walls if the swarm traveled down the center, but there were choke points and traps which could slow the participant down. The participant was instructed that the optimal strategy is to avoid the traps. Single digit addition problems blocked the viewport randomly every ten to fifteen seconds until a correct answer is given, leaving only the side panel with the Force Feedback and Input vectors visible. Normal operation occurred behind the math problem. This condition corresponds to a scenario where navigation is a secondary task and an infrequent primary task may require full visual attention, i.e. checking video surveillance while directing the swarm.

The Speed environment is structured around many decisions, see Figure 4(b). The dead ends and intersections require focus and decision-making for operators to determine the best path to explore as much area as possible in order to find as many of the targets as possible. As with the corridor environments, moving down the center of the doors or corridors with a large portion of the swarm should cover both sides. The distinctive feature of this environment is 
(a)

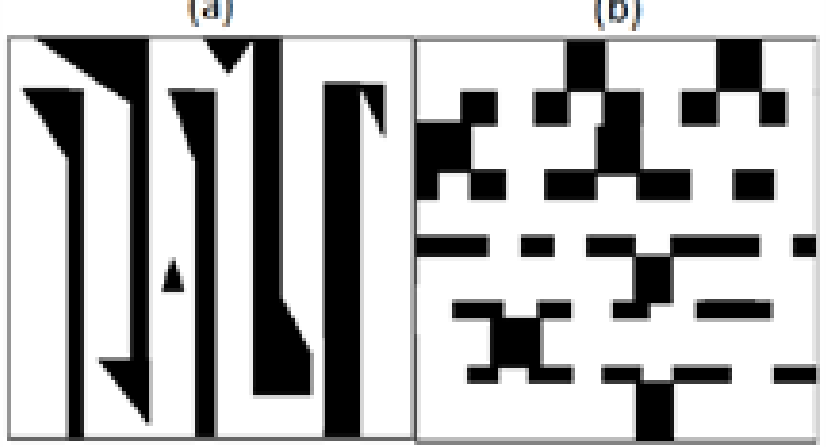

(c)

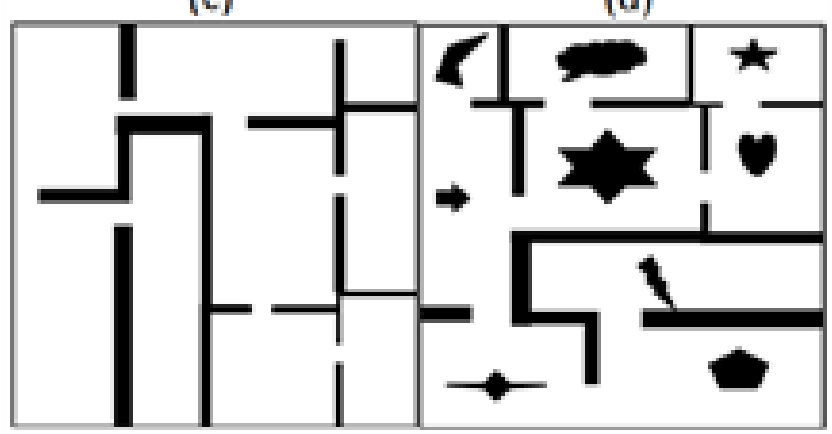

Fig. 4. Four environments used in the study, the robots always started in the lower left corner: (a) Math, (b) Speed, (c) Control, and (d) Hidden.

greater speed, which requires changes in the force constants to avoid wall collisions. The speed was set to $1.0 \mathrm{~m} / \mathrm{s}$ and the constants were set to $k_{o}=10, k_{r}=4, k_{h}=5$. All other constants remained the same. This created a volatile swarm which was more likely to break up around obstacles. The participants were warned of this.

The Control environment is a structured office type environment without obstacles in the rooms, see Figure 4(c). This was used as the control world without special features. Participants were told to explore the rooms thoroughly before moving to the next one to efficiently explore the area. This was the optimal strategy because traversing doorways slows the swarm down and spreads them out, requiring extra attention to maintain redundant coverage to mark targets.

The Hidden environment is structured with obstacles with edges and concave corners requiring exploration, see Figure 4(d). In this environment, the obstacles are hidden, so the participants had no idea where the obstacles were, except by observed behavior, side panel information, and haptic feedback (if in the haptic condition). The participants were told it was an office structure with obstacles in the room, and that the best strategy was to sweep the rooms avoiding a lot of force from the walls and then finding the exit, similar to the strategy in the Control environment. They were also instructed to use marked targets as landmarks when doorways were thought to be found in case the room was a dead end.

\section{Experimental Design}

This paper uses a user study with a between-subjects design. Thirty-two paid student participants from the University of Pittsburgh were divided into two groups. One group received visual and physical force feedback in the Phantom device as described above, hereafter referred to as the haptics group. The control group received only visual feedback, although the force panels conveyed all of the same information that the haptics group received, see Fig. 1. All other variables remained constant. Participants were given explanations of the robot motion algorithms, the interface, the importance of a cohesive swarm, and were given 10 minutes of practice to gain experience and ask questions. A math problem popped up every thirty to sixty seconds to help prepare for the Math condition. Environments were presented in random order. The participants were given fifteen minutes in each environment to find as many targets as possible. After each environment, the participants were given a brief questionnaire to help determine the difficulty and frustration of the task and helpfulness and frequency of use of the different panels using a seven level Likert scale.

\section{RESULTS}

The data shows results for environment coverage of the threshold required for marking targets, and some possible explanations found in the questionnaire data. Comparisons between conditions and environments were tested with ANOVAs. Results $\mathrm{p}<0.05$ are considered significant and $\mathrm{p}<0.015$ are considered marginally significant. To measure the participants' performance and compare the utility of the novel method of returning haptics feedback to the operator, percent of traversable map coverage is used. Due to the redundancy required to find targets, only parts of the map sensed by five or more robots simultaneously was considered covered. This measurement helps remove the luck factor of finding targets from [17].

The findings show an improvement in this new performance measure of haptic feedback when comparing all environments except the Hidden environment $(\mathrm{p}<0.01)$, see Fig. 5. These results show that in these environments, participants were able to better control the swarm in order to cover more area during the fifteen minutes allotted to find targets. This result shows that either the participants were able to keep the swarm together better to cover more area for target marking or they more efficient traversed the corridors, doorways, and other obstacles in order to cover more ground during the fifteen minute trial period. The questionnaire points to a possible contributing factor of the haptic feedback over the control condition. One question asked how helpful the force feedback panel display was on a scale from 1-7. Fig. 6 shows that those in the haptic condition thought the force feedback panel more useful $(\mathrm{p}<0.03)$. The higher rating from haptic participants shows that the force in the device help make the forces show on the display more intuitive and allows the participant to better use the information to accomplish the task (i.e. staying in the center of corridors or 


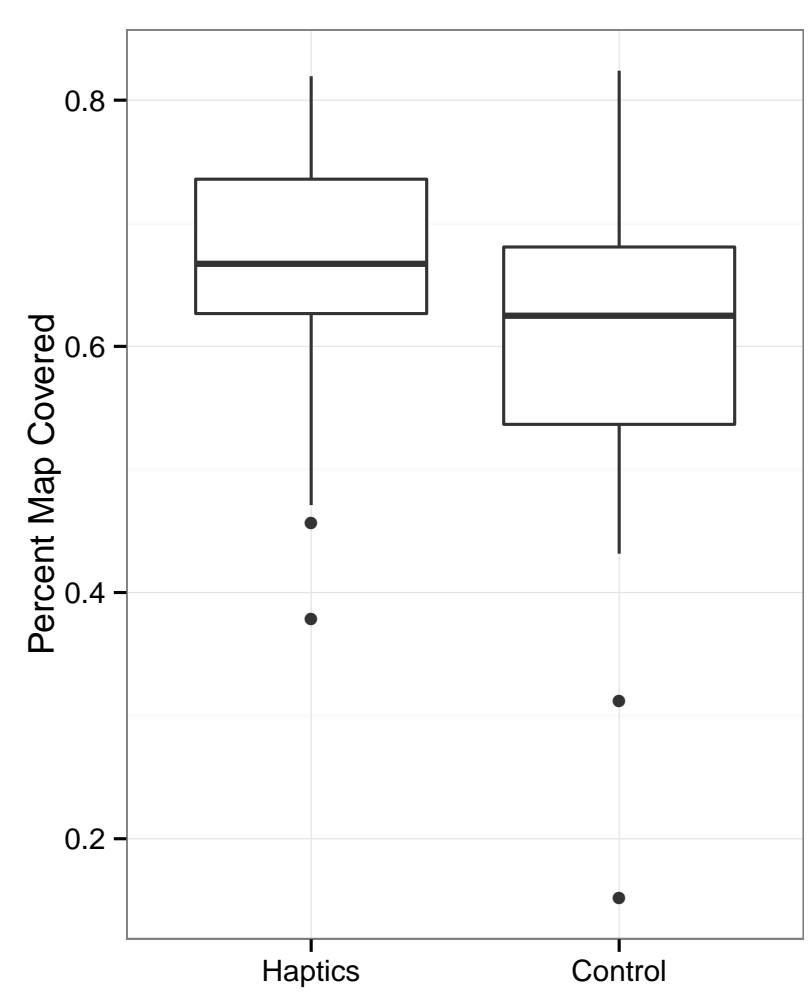

Fig. 5. Box plot around the median percent of the map covered in each trial of all environments except for Hidden between conditions. Each box represents 48 trials.

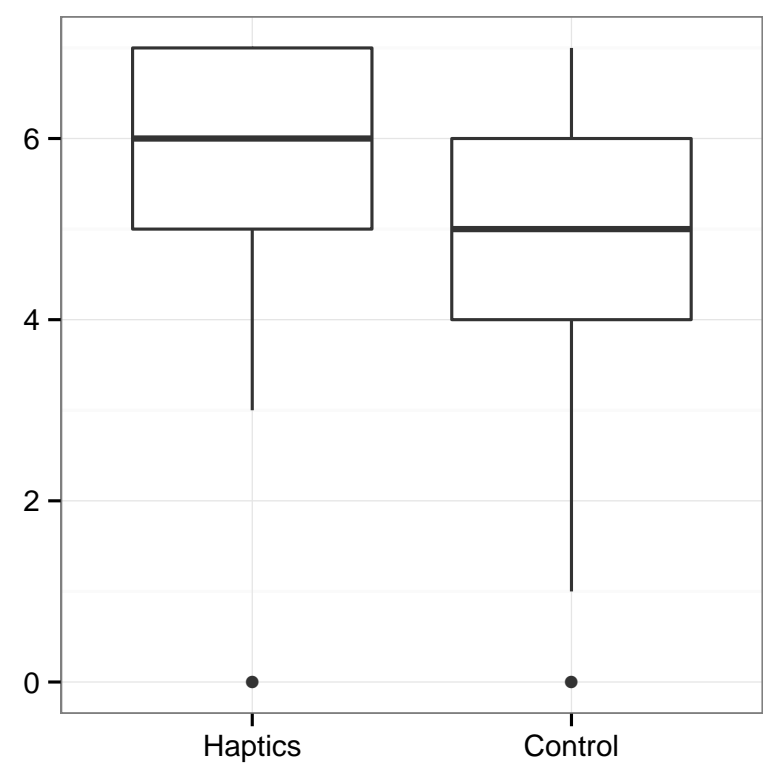

Fig. 6. Box plot around the median value for the question asking the utility of the force feedback panel display between conditions. Each box represents 64 trials. going through the middle of doorways to maximize efficient traversal).

When separating out the environments, the performance measure shows a marginal improvement in coverage for the haptics condition in the Math, Speed, and Control environments ( $\mathrm{p}<0.15, \mathrm{p}<0.08$, and $\mathrm{p}<0.08$ respectively), as shown in Fig. 7. This does not hold for the Hidden environment $(\mathrm{p}<0.55)$. The first two questions in the questionnaire asked about the difficulty and the level of frustration of the task in each environment respectively. The hidden environment proved to be more difficult and more frustrating than any other environment based on this questionnaire $(\mathrm{p}<0.001)$. The level of frustration shows that the intuition or strategy to efficiently accomplish the task was not obvious and participants were unable to take advantage of the information from the screen or through the haptic device to help accomplish the task in this type of environment. Further work is necessary to try and determine a training method or interface change that could help the participants perform better in this type of environment in order to determine whether or not haptic feedback is advantageous.

\section{CONCLUSIONS}

This paper introduced a novel method of giving operators force feedback from a group of decentralized robots and their environment to allow for more efficient environment coverage given the control structure described above. The user study results show that the haptic feedback helps the participants navigate around obstacles more efficiently than participants in the same scenario without any haptic feedback, except the very difficult Hidden environment. Even in the Hidden environment, the haptic feedback did not have a detrimental effect on the performance, so within the bounds of this study, no negative effect from haptic feedback, given in this manner, is found. It appears that when the position of obstacles and the robots are known, the haptic feedback helps operators maintain a cohesive swarm while they explore the environment in search of targets. In real applications this could mean finding victims of disasters scenarios faster in order to get them the aid they need.

Future studies should further explore the Hidden environment. The more intuitive means of information given through the haptic feedback should help operators navigate through unknown environments just as it does in the other environments, but the results prove otherwise. As noted above, better training or interface clues may help operators use the information more effectively and explore the area better in this scenario of fewer assumptions. Also, this is just the first look of haptic feedback in a human swarm interactive system. Other methods, controls, and environments should be tried in order to maximize the feedback's utility. For instance, maybe there is a better way to aggregate the haptic information besides averaging it across the swarm (i.e. only averaging swarm members who are interacting with an obstacle). This might allow operators to notice obstacle interaction sooner and respond more efficiently. Finally, the current structure assumes perfect state information of robots 

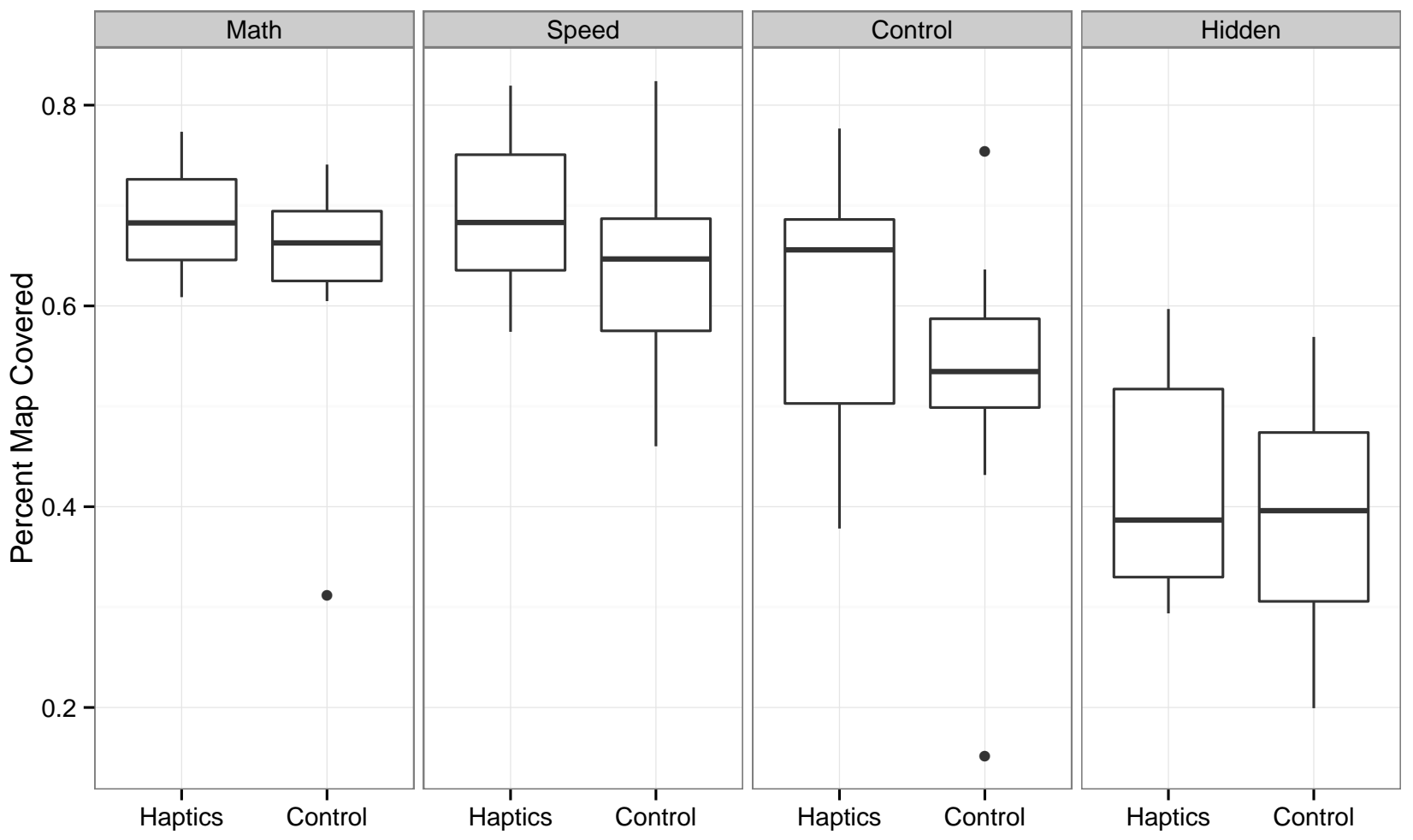

Fig. 7. Box plot around the median percent of the map covered in each trial of each environment between conditions. Each box represents 8 trials.

and the intent of the operator. These assumptions often do not hold, so this method must be tested with error to overcome the problems likely in real world scenarios.

\section{REFERENCES}

[1] Z. Kira and M. Potter, "Exerting human control over decentralized robot swarms," in Autonomous Robots and Agents, 2009. ICARA 2009. 4th International Conference on. IEEE, 2009, pp. 566-571.

[2] S. Bashyal and G. Venayagamoorthy, "Human swarm interaction for radiation source search and localization," in Swarm Intelligence Symposium, 2008. SIS 2008. IEEE. IEEE, 2008, pp. 1-8.

[3] M. Fields, E. Haas, S. Hill, C. Stachowiak, and L. Barnes, "Effective robot team control methodologies for battlefield applications," in Intelligent Robots and Systems, 2009. IROS 2009. IEEE/RSJ International Conference on. IEEE, 2009, pp. 5862-5867.

[4] A. Naghsh, J. Gancet, A. Tanoto, and C. Roast, "Analysis and design of human-robot swarm interaction in firefighting," in Robot and Human Interactive Communication, 2008. RO-MAN 2008. The 17th IEEE International Symposium on. IEEE, 2008, pp. 255-260.

[5] A. Kolling, S. Nunnally, and M. Lewis, "Towards human control of robot swarms," in Proceedings of the 7th international conference on Human-robot interaction. ACM, 2012, pp. 89-96.

[6] S. Nunnally, P. Walker, A. Kolling, N. Chakraborty, M. Lewis, K. Sycara, and M. Goodrich, "Human influence of robotic swarms with bandwidth and localization issues," Systems, Man, and Cybernetics (SMC), 2012 IEEE International Conference on, pp. 333-338, 2012.

[7] M. Goodrich, B. Pendleton, P. Sujit, and J. Pinto, "Toward human interaction with bio-inspired robot teams," in Systems, Man, and Cybernetics (SMC), 2011 IEEE International Conference on. IEEE, 2011, pp. 2859-2864.

[8] P. Klarer, "Flocking small smart machines: An experiment in cooperative, multi-machine control," Sandia National Labs., Albuquerque, NM (United States), Tech. Rep., 1998.

[9] M. Cummings, "Human supervisory control of swarming networks," in 2nd Annual Swarming: Autonomous Intelligent Networked Systems Conference, 2004.
[10] G. Coppin and F. Legras, "Autonomy spectrum and performance perception issues in swarm supervisory control," Proceedings of the IEEE, no. 99, pp. 590-603, 2012.

[11] C. Secchi, A. Franchi, H. Bülthoff, and P. Giordano, "Bilateral teleoperation of a group of uavs with communication delays and switching topology," Proceedings of the IEEE International Conference on Robotics and Automation, 2012.

[12] A. Franchi, P. R. Giordano, C. Secchi, H. I. Son, and H. H. Bulthoff, "A passivity-based decentralized approach for the bilateral teleoperation of a group of uavs with switching topology," in Robotics and Automation (ICRA), 2011 IEEE International Conference on. IEEE, 2011, pp. 898-905.

[13] E. J. Rodríguez-Seda, J. J. Troy, C. A. Erignac, P. Murray, D. M. Stipanovic, and M. W. Spong, "Bilateral teleoperation of multiple mobile agents: Coordinated motion and collision avoidance," Control Systems Technology, IEEE Transactions on, vol. 18, no. 4, pp. 984992, 2010.

[14] D. Lee, A. Franchi, P. Giordano, H. Son, and H. Bulthoff, "Haptic teleoperation of multiple unmanned aerial vehicles over the internet," in Robotics and Automation (ICRA), 2011 IEEE International Conference on. IEEE, 2011, pp. 1341-1347.

[15] A. Howard, M. Matarić, and G. Sukhatme, "An incremental selfdeployment algorithm for mobile sensor networks," Autonomous Robots, vol. 13, no. 2, pp. 113-126, 2002.

[16] S. Nunnally, N. Chakraborty, P. Walker, M. Lewis, and K. Sycara, "Using haptics in human robotic swarm interaction," in International Annual Meeting of the Human Factors and Ergonomics Society, 2013.

[17] B. Gerkey, R. Vaughan, and A. Howard, "The player/stage project: Tools for multi-robot and distributed sensor systems," in Proceedings of the 11th international conference on advanced robotics. Portugal, 2003, pp. 317-323.

[18] M. Quigley, K. Conley, B. Gerkey, J. Faust, T. Foote, J. Leibs, R. Wheeler, and A. Ng, "Ros: an open-source robot operating system," in ICRA Workshop on Open Source Software, vol. 3, no. 3.2, 2009. 